TOKYo J. Math.

Vol. 15 , No. 2, 1992

\title{
On the Galois Group of $x^{p}+p^{t} b(x+1)=0$
}

\section{Kenzo KOMATSU}

Keio University

1. In [3] we discussed the Galois group of

$$
x^{p}+a x+a=0
$$

over the rational number field $Q$, where $p$ is a prime number, and $a \in Z,(p, a)=1$. The situation becomes much more complicated when $a$ is divisible by $p$. In this paper we deal with three special cases:

1. $a=p^{t} b, 0<t<p,(p, b)=1,\left|(p-1)^{p-1} b+p^{p-t}\right|$ is not a square;

2. $a=p k^{2},(p, k)=1$;

3. $a=p^{2 m} b, 0<2 m<p,(p, b)=1$.

We begin by proving the following theorem (cf. [3]).

THEOREM 1. Let $a_{0}, a_{1}, \cdots, a_{n-1}$ be rational integers such that

$$
f(x)=x^{n}+a_{n-1} x^{n-1}+\cdots+a_{1} x+a_{0}
$$

is irreducible over the rational number field $Q$. Let $\alpha$ be a root of $f(x)=0$, and let

$$
\begin{gathered}
\delta=f^{\prime}(\alpha), \quad D=\operatorname{norm} \delta(\text { in } Q(\alpha)), \\
D / \delta=x_{0}+x_{1} \alpha+\cdots+x_{n-1} \alpha^{n-1}, \quad x_{i} \in Z .
\end{gathered}
$$

Let $D_{1}$ and $D_{2}$ denote any rational integers which satisfy the following conditions:

$$
\begin{gathered}
D=D_{1} D_{2}, \\
\left(D_{1}, D_{2}\right)=1, \\
\left(D_{2}, x_{0}, x_{1}, \cdots, x_{n-1}\right)=1 .
\end{gathered}
$$

Let $G$ denote the Galois group of $f(x)=0$ over $Q ; G$ is a transitive permutation group on the set $\{1,2, \cdots, n\}$. Then we have:

I. If $\left|D_{2}\right|$ is not a square, $G$ contains a transposition.

II. If $\left|D_{2}\right|$ is a square, $D_{1}$ is divisible by the discriminant of $Q(\alpha)$.

Proof. Suppose first that $\left|D_{2}\right|$ is not a square. Then there exists a prime number 
$q$ such that $\left(D_{2}\right)_{q}$ is odd, where the symbol $\left(D_{2}\right)_{q}$ means the largest integer $M$ such that $D_{2}$ is divisible by $q^{M}$ (cf. [1]). Since $D_{2}$ is divisible by $q$, it follows from (1.3) that $q \nmid x_{i}$ for some $i$. Clearly, $(D)_{q}$ is also odd. Hence, by Theorem 1 of [1], we see that the discriminant $d$ of $Q(\alpha)$ is exactly divisible by $q$. Therefore $G$ contains a transposition ([4]). Suppose next that $\left|D_{2}\right|$ is a square. Let $q$ denote a prime factor of $D_{2}$. Then, by (1.3), we see that $q \nmid x_{i}$ for some $i$. Since $(D)_{q}=\left(D_{2}\right)_{q}$ is even, it follows from Theorem 1 of [1] that $d$ is not divisible by $q$. Hence we obtain $\left(d, D_{2}\right)=1$. Since $D$ is divisible by $d$, we see that $D_{1}$ is divisible by $d$.

2. Now we prove the following theorem.

THEOREM 2. Let $p$ denote an odd prime, and let $t$ and $b$ denote rational integers such that $0<t<p,(p, b)=1$. Suppose that $\left|(p-1)^{p-1} b+p^{p-t}\right|$ is not a square. Then the Galois group of

$$
x^{p}+p^{t} b(x+1)=0
$$

over $\boldsymbol{Q}$ is the symmetric group $S_{p}$.

ProOf. Since $0<t<p, t$ is not divisible by $p$. It is easily seen that

$$
f(x)=x^{p}+p^{t} b(x+1)
$$

is irreducible over $\boldsymbol{Q}$ ([2], Lemma 1). Let $\alpha$ be a root of $f(x)=0$, and let $\delta=f^{\prime}(\alpha)$, $D=\operatorname{norm} \delta$ (in $Q(\alpha))$. Then ([1], Theorem 2)

$$
\begin{aligned}
D & =(p-1)^{p-1}\left(p^{t} b\right)^{p}+p^{p}\left(p^{t} b\right)^{p-1} \\
& =p^{t p} b^{p-1}\left\{(p-1)^{p-1} b+p^{p-t}\right\} .
\end{aligned}
$$

Now let

$$
D_{1}=p^{t p} b^{p-1}, \quad D_{2}=(p-1)^{p-1} b+p^{p-t}
$$

Then

$$
D=D_{1} D_{2}, \quad\left(D_{1}, D_{2}\right)=1 .
$$

By Theorem 2 of [1] we see that the condition (1:3) of Theorem 1 is also satisfied. Since $p$ is a prime, the Galois group of $f(x)=0$ is primitive. Theorem 1 implies that the Galois group is the symmetric group $S_{p}$ ([5], Theorem 13.3).

3. Consider now the case

$$
a=p k^{2}, \quad(p, k)=1 .
$$

From Theorem 2 we obtain 
THEOREM 3. Let $p$ denote a prime number, and $k$ a rational integer such that $(p, k)=1$. Then the Galois group of

$$
x^{p}+p k^{2}(x+1)=0
$$

over $Q$ is the symmetric group $S_{p}$.

Proof. We may assume that $p>2, k>0$. When $p=3$, the Galois group of (3.1) is the symmetric group $S_{3}$, since the discriminant of (3.1) is negative. So we may assume that

$$
p>3, \quad k>0 \text {. }
$$

Now suppose that

$$
(p-1)^{p-1} k^{2} \hbar p^{p-1}=c^{2}, \quad c \in Z, \quad c>0 .
$$

Then we have

$$
\begin{aligned}
p^{p-1} & =c^{2}-(p-1)^{p-1} k^{2} \\
& =\left\{c-(p-1)^{(p-1) / 2} k\right\}\left\{c+(p-1)^{(p-1) / 2} k\right\} .
\end{aligned}
$$

Clearly,

$$
c+(p-1)^{(p-1) / 2} k
$$

is positive, and prime to

$$
c-(p-1)^{(p-1) / 2} k
$$

Hence

$$
c+(p-1)^{(p-1) / 2} k=p^{p-1}, \quad c-(p-1)^{(p-1) / 2} k=1
$$

Therefore

$$
p^{p-1}-1=2 k(p-1)^{(p-1) / 2},
$$

and so

$$
k=\frac{p^{p-1}-1}{2(p-1)^{(p-1) / 2}}
$$

Now let

$$
\frac{p-1}{2}=B
$$

so that

$$
p-1=2 B, \quad p=2 B+1 \text {. }
$$


Then (3.4) becomes

$$
k=\frac{(2 B+1)^{2 B}-1}{2(2 B)^{B}} .
$$

Since $p>3$, we have $B \geq 2$. When $B=2$, (3.5) gives

$$
k=\frac{5^{4}-1}{2 \cdot 4^{2}},
$$

which is not an integer. So we may assume that $B \geq 3$. Then, by (3.5) we see that

$$
\frac{(2 B+1)^{2 B}-1}{(2 B)^{3}}
$$

is an integer. On the other hand,

$$
\begin{aligned}
(2 B+1)^{2 B}-1 & =(2 B)^{2 B}+\cdots+\frac{(2 B)(2 B-1)}{2}(2 B)^{2}+(2 B)(2 B) \\
& \equiv(2 B)^{2}\left(2 B^{2}-B+1\right) \quad\left(\bmod (2 B)^{3}\right) .
\end{aligned}
$$

Hence $(2 B+1)^{2 B}-1$ is not divisible by $(2 B)^{3}$.

A contradiction shows that

$$
(p-1)^{p-1} k^{2}+p^{p-1}
$$

is not a square. By Theorem 2 we see that the Galois group of (3.1) over $Q$ is the symmetric group $S_{p}$.

As a special case $(k=1)$ of Theorem 3, we obtain

THEOREM 4. For any prime number $p$, the Galois group of

$$
x^{p}+p x+p=0
$$

over $Q$ is the symmetric group $S_{p}$.

4. Now we discuss the case

$$
a=p^{2 m} b, \quad 0<2 m<p, \quad(p, b)=1 .
$$

THEOREM 5. Let $p(p>3)$ denote a prime number and let $b$ and $m$ denote rational integers such that $0<2 m<p,(p, b)=1$. Let $G$ denote the Galois group of the equation

$$
x^{p}+p^{2 m} b(x+1)=0
$$

over $\boldsymbol{Q}$.

1. If $p \equiv 3$ or 5 or $7(\bmod 8)$, then $G$ is the symmetric group $S_{p}$. 
2. Suppose that $p \equiv 1(\bmod 8)$. Then $G=S_{p}$ if and only if $(p-1)^{p-1} b+p^{p-2 m}$ is not a square. If $(p-1)^{p-1} b+p^{p-2 m}$ is a square, then $G$ is contained in the alternating group $A_{p}$, where $G$ is regarded as a permutation group on $\{1,2, \cdots, p\}$.

Proof. We have

$$
p^{p-2 m} \equiv p \quad(\bmod 8) .
$$

Also, for every prime factor $q$ of $p-1$,

$$
p^{p-2 m} \equiv 1 \quad(\bmod q) .
$$

If $p \equiv 3$ or 5 or $7(\bmod 8)$, then

$$
\left|(p-1)^{p-1} b+p^{p-2 m}\right|
$$

is not a square ([3], the proof of Theorem 1), and so $G=S_{p}$ (Theorem 2).

Now suppose that $p \equiv 1(\bmod 8)$. It follows from $(4.1)$ that $-\left\{(p-1)^{p-1} b+p^{p-2 m}\right\}$ is not a square. Hence, if $(p-1)^{p-1} b+p^{p-2 m}$ is not a square, then $G=S_{p}$ (Theorem 2). Suppose further that $(p-1)^{p-1} b+p^{p-2 m}$ is a square. Let $\alpha_{1}, \alpha_{2}, \cdots, \alpha_{p}$ denote the roots of

$$
f(x)=x^{p}+p^{2 m} b(x+1)=0,
$$

and let $\delta=f^{\prime}\left(\alpha_{1}\right), D=\operatorname{norm} \delta$ (in $Q\left(\alpha_{1}\right)$ ). Then, by (2.1) we see that $D$ is also a square. Now let $A$ denote the following matrix:

$$
A=\left(a_{i j}\right), \quad a_{i j}=\alpha_{i}^{j-1}(1 \leq i \leq p ; 1 \leq j \leq p) .
$$

Then we have

$$
(\operatorname{det} A)^{2}=(-1)^{p(p-1) / 2} D=D .
$$

Hence $\operatorname{det} A$ is a rational integer. If $g \in G$ is an odd permutation, then

$$
(\operatorname{det} A)^{g}=-(\operatorname{det} A) \text {, }
$$

which is impossible. Hence $G$ is contained in $A_{p}$.

Finally we prove

THEOREM 6. For any prime number $p \equiv 1(\bmod 8)$ and any rational integer $m$ with $0<2 m<p$, there exist infinitely many rational integers $b$ satisfying the following conditions:

1. $(p, b)=1$;

2. $(p-1)^{p-1} b+p^{p-2 m}$ is a square.

Proof. The congruence

$$
x^{2} \equiv p^{p-2 m} \quad\left(\bmod (p-1)^{p-1}\right)
$$

has a solution $x((4.1),(4.2))$. We may assume that $x$ is not divisible by $p$, since 
$x+(p-1)^{p-1}$ is also a solution of (4.3). Now let

$$
x^{2}-p^{p-2 m}=y(p-1)^{p-1} .
$$

Then $y$ is not divisible by $p$. For every $n \in Z$,

$$
b=y+2 x n p+n^{2} p^{2}(p-1)^{p-1}
$$

satisfies the conditions of Theorem 6 , since

$$
(p-1)^{p-1} b+p^{p-2 m}=\left(x+n p(p-1)^{p-1}\right)^{2} .
$$

\section{References}

[ 1 ] K. Komatsu, Integral bases in algebraic number fields, J. Reine Angew. Math., 278/279 (1975), 137144.

[ 2 ] K. Komatsu, On certain homogeneous Diophantine equations of degree $n(n-1)$, Tokyo J. Math., 12 (1989), 231-234.

[ 3 ] K. KomatSU, On the Galois group of $x^{p}+a x+a=0$, Tokyo J. Math., 14 (1991), 227-229.

[4] B. L. VAN DER WAERden, Die Zerlegungs- und Trägheitsgruppe als Permutationsgruppen, Math. Ann., 111 (1935), 731-733.

[ 5 ] H. Wieland, Finite Permutation Groups, Academic Press, 1964.

Present Address:

Department of Mathematics, Faculty of Science and Technology, Keio University HiYoshi, KoHOKU-KU, YOKOHAMA 223, JAPAN 